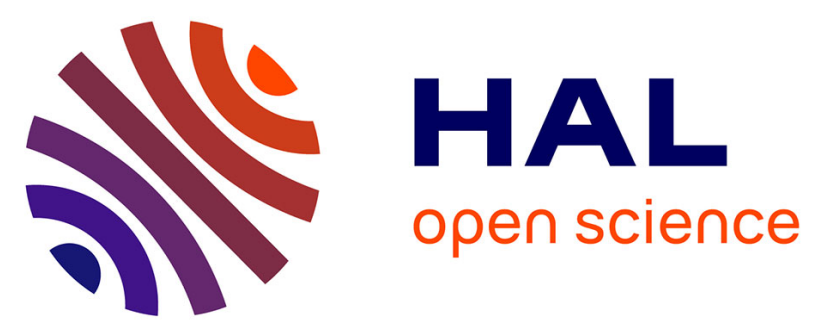

\title{
An Ontological Model for Supporting Intention-Based Information Sharing on Collaborative Problem Solving
}

Rodrigo Bonacin, Julio Cesar dos Reis, Heiko Hornung, Maria Cecilia Calani Baranauskas

\section{- To cite this version:}

Rodrigo Bonacin, Julio Cesar dos Reis, Heiko Hornung, Maria Cecilia Calani Baranauskas. An Ontological Model for Supporting Intention-Based Information Sharing on Collaborative Problem Solving. International Journal of Collaborative Enterprise (IJCEnt), 2013, 3 (2/3), pp.130-150. hal-00833713

\section{HAL Id: hal-00833713 https://hal.science/hal-00833713}

Submitted on 13 Jun 2013

HAL is a multi-disciplinary open access archive for the deposit and dissemination of scientific research documents, whether they are published or not. The documents may come from teaching and research institutions in France or abroad, or from public or private research centers.
L'archive ouverte pluridisciplinaire HAL, est destinée au dépôt et à la diffusion de documents scientifiques de niveau recherche, publiés ou non, émanant des établissements d'enseignement et de recherche français ou étrangers, des laboratoires publics ou privés. 


\title{
An Ontological Model for Supporting Intention-Based Information Sharing on Collaborative Problem Solving
}

\author{
Rodrigo Bonacin \\ CTI Renato Archer and Faculty of Campo Limpo Paulista (FACCAMP) \\ Rodovia Dom Pedro I, Km 143.6 - 13069-901 \\ Campinas, SP - Brazil \\ rodrigo.bonacin@cti.gov.br
}

\section{Julio Cesar Dos Reis}

CR SANTEC - Public Research Centre Henri Tudor and LRI, University of Paris-Sud XI Av. des hauts fourneaux, L-4362

Esch-sur-Alzette - Luxembourg

julio.dosreis@tudor.lu

\author{
Heiko Hornung, M. Cecília C. Baranauskas \\ Institute of Computing - UNICAMP \\ Av. Albert Einstein $\mathrm{n}^{\circ} 1251$, \\ 13084-722 \\ Campinas, SP - Brazil \\ heix@gmx.com, cecilia@ic.unicamp.br
}

\begin{abstract}
Web-based collaborative systems are potentially a rich source of knowledge for lifelong learning and professional development. In these systems, practitioners can share messages about their problems on daily work activities. These messages can also be used for analytical purposes, e.g., for understanding the development of a discussion as well as for evaluating how a system supports different types of conversations. These possibilities depend on a proper classification and adequate recovery of the messages regarding semantic and pragmatic aspects. The construction of a knowledge representation model that considers the pragmatic aspects of the messages is still an open research challenge. Such a model might support an intention-based retrieval of messages. In this investigation we argue that a model of this nature must include the association of semantics with illocutionary aspects of the users' communication. This paper proposes an ontology using Semantic Web standards to associate messages to illocutions and ontology terms, as an integrated representation. The proposed model is grounded in the Pragmatic Web perspective including Semiotics and Speech Act Theory. Furthermore, we conduct a case study with scenarios of two different systems, where special education specialists share problems of their daily work activities.
\end{abstract}

Keywords: Pragmatics; Collaborative Systems; Knowledge Representation; Problem Solving; Semantic and Pragmatic Web

Biographical notes: Rodrigo Bonacin is a Senior Researcher at CTI Renato Archer, Brazil and FACCAMP, Brazil. He received a B.Sc. and M.Sc. in Computer Science at UFPR, Brazil and a Ph.D. in Computer Science at UNICAMP, Brazil, and Postdoctorate by Public Research Centre Henri Tudor, Luxembourg. His research interests include Ontologies, Human ComputerInteraction, Medical Informatics and Organizational Semiotics. 
Julio Cesar Dos Reis is a Research Assistant at CRP Henri Tudor (Luxembourg) and Ph.D. Candidate in Computer Science at University of Paris-Sud XI (France). In 2011 he obtained a M.Sc. degree in Computer Science at University of Campinas (Brazil). Julio's research interest focuses mainly on the areas of Knowledge Engineering and Human-Computer Interaction. He investigates topics related to knowledge representation, evolution, integration, retrieval and visualization taking semantic, pragmatic and social aspects into account.

Heiko Hornung is currently a $\mathrm{PhD}$ candidate at the Institute of Computing of the University of Campinas, UNICAMP, Brazil. He holds a degree ("Diplom") in business informatics from Darmstadt University of Technology, Germany, and a master's degree in computer science from UNICAMP. His research interests span topics such as e-government, e-inclusion, interaction design, electronically-mediated human-human interaction, and universal access to information and knowledge.

M. Cecilia C. Baranauskas is a Brazilian Professor and researcher in Human-Computer Interaction (HCI) at the University of Campinas (UNICAMP). She received the ACM Rigo Award (2010) for her lifetime contribution to the Design of Communication field, was entitled Honorary Research Fellow at the Staffordshire University (2001) and Visiting Fellow at the University of Reading (UK). She has led several projects in the context of e-Citizenship and e-Inclusion, and has advised more than 40 Master dissertations and $\mathrm{PhD}$ theses. Her research interests focus on HCI issues, particularly investigating different formalisms in the analysis, design and evaluation of societal systems.

\section{Introduction}

The Web has a great potential to promote dynamic environments for collaborative discussion and problem solving. The Semantic Web (SemWeb) proposes to model the knowledge using Web ontologies, with the aim of producing a better understanding and interpretation of Web content by humans and computational agents. However, the design of mechanisms with the focus on who will use the SemWeb technology is still an open issue for the SemWeb research community. This is particularly important in the development of systems that intend to support users in sharing professional experiences and promoting lifelong learning. In this paper, we argue that pragmatics, e.g. people's intentions and the context in which language is used, is a key factor to be considered in encouraging meaningful information sharing.

According to Hendler and Berners-Lee (2010) "The basic Semantic Web technologies have been defined and are starting to be more widely deployed with further components of the architecture being the focus of current standardization efforts. However, there has still been very little work in understanding the impact of this new capability: how it truly enables the connections of the Web of people who will use it." They also expose the challenge of turning messy human knowledge into a shared information space that is useful to everyone.

In the context of the collaborative Web, knowledge representation models could enable richer user interactions and possibilities by considering the evolution of the pragmatic aspects and their relations with the semantic ones. In problem solving, for instance, usually the interactions, rationale and history of the actions are as important as (or even more important than) the solution itself. The interpretation of content generated during a collaborative process (e.g., 
messages, discussions, documents) is very dependent on the analysis of the author's intention at the time when it was created. A problem still to be explored is how to associate adequate communication theories and methods with a computational model able to represent aspects related to intentionality of messages in problem solving. Once represented, aspects related to intentions can be explored in richer scenarios where users want to share, interpret or recover information from the messages.

In this paper, we present an ontology described in Web Ontology Language (OWL) as a knowledge model to represent the relation between intentions and meaning in the context of collaborative problem solving messages. The modeling process and fundamentals adopted in the proposed approach have their roots in Pragmatic Web (PragWeb) research.

Originally proposed as an extension or a complement of the SemWeb, the PragWeb addresses topics such as context and meaning negotiation in the Web (Singh, 2002; Schoop et al., 2006). Moreover, studies including Speech Act Theory (SAT) (Austin, 1962; Searle, 1976) and Organizational Semiotics (OS) (Liu, 2000) provide useful and important methods to identify and quantify aspects related to intentions (and pragmatics in general) of communication acts. Various PragWeb studies are grounded in these theoretical bases. One of these methods is the Pragmatics Communication Analysis presented by Liu (2000).

This paper contributes with an OWL model to represent and associate results of the Pragmatics Communication Analysis with external computational models. The main objective is to explore new possibilities of interaction and information sharing based on the model for providing meaningful web collaborations.

The model is inspired by two previous empirical studies (Bonacin et al., 2012) of problem solving scenarios: the first in the VilaNaRede Social Network System $^{1}$, which adopts a forum structure for questions and discussions, and the second within Yahoo! Answers ${ }^{\circledR}{ }^{2}$, which adopts the structure of answers towards a unique posted question. These previous studies were used to investigate and explore interaction possibilities regarding intentionality in problem solving, as well as to elicit requirements for the model proposed in this paper.

This paper also presents scenarios from these empirical studies that explore the proposed model to improve information retrieval. In these scenarios, results of possible user queries are confronted with results of syntactic/lexical and semantic approach. Particularly, the objective is to explore new possibilities brought by the model, as well as its limitations.

The paper is organized as follows: Section II presents the background in the PragWeb field and Pragmatics Communication Analysis; Section III briefly describes and summarizes results from previous studies, which give empirical support for the adopted modeling approach; Section IV presents the proposed OWL ontology model; Section V presents the conducted study while Section VI discusses the results and related work; Finally, Section VII concludes and indicates future work.

\footnotetext{
${ }^{1}$ www.vilanarede.org.br

${ }^{2} \mathrm{http}$ ://answers.yahoo.com
} 


\section{Background}

This section presents the background work in PragWeb and the Pragmatics Communication Analysis, which were used as a methodological frame of reference for the studies and the proposed ontology model.

\section{A. The Pragmatic Web and its foundations}

The PragWeb is concerned with questions such as how knowledge is actually constructed, and how it evolves during the collaboration among people, mediated by Web artifacts. The concept emerged to address some critical issues of the SemWeb, e.g., the complexity of content creation and maintenance (McCool, 2005), and the underestimated consideration of context (Singh, 2002). The PragWeb perspective has been applied to a variety of research domains, e.g., multi-agent systems (Paschke et al., 2007), interaction design (Hornung and Baranauskas, 2011), self-organizing communities of practice (de Moor and van den Heuvel, 2004), as well as Web Services (Liu, 2009).

PragWeb research is rooted in different Information Systems research frameworks and theories, e.g., the Language/Action Perspective (LAP) (Goldkuhl and Lyytinen, 1982; Winograd and Flores, 1987) or OS (Liu, 2000). The basic unit of analysis of LAP is a speech act (from SAT). LAP subscribes to the notion that we perform actions through language. Thus, collaboration is coordinated by the performance of speech acts, which underlie socially determined rules (Schoop et al, 2006).

OS is a branch of Semiotics that understands and investigates organizations as systems of signs. OS studies the nature, characteristics, functions and effects of information and communication in organizational contexts. An organization is considered a social system in which people behave in an organized manner, and in which organizational behavior is shaped by a system of norms as well as by people's individual or joint communication and interpretation of signs (Liu, 2000). In OS, basic units of analysis are affordances and agents. Initially introduced by Gibson (1968), the concept of affordances was expanded by Stamper (1996) to represent patterns of behavior that are governed by systems of norms in the physical and social world. Agents are entities (persons or groups of people) that can be attributed with responsibility. OS's basic ideas have been formulated as "there is no knowledge without a knower, and there is no knowing without action" (Liu, 2000 p. 26). In addition, OS subscribes to the notion that knowledge about the world, and the underlying systems of norms are constantly changing.

With LAP and OS (Cordeiro and Filipe, 2003) as possible theoretic frames of reference, and with Web-mediated collaboration and meaning negotiation of people under the condition of change and evolution as its object of study, the Pragmatic Web thus provides an important basis for this work. LAP and OS were also adopted as background for the analysis method described in the next section. 
Title

\section{B. Pragmatics Communication Analysis}

According to Morris (1938) Pragmatics can be understood as the relationship between signs and humans. It concerns aspects such as intentions, communications, conversations and negotiations (Liu, 2000; Stamper, 1996).

In LAP, language is understood as action, and SAT provides us the theory for studying language and the use of language in human communication. According to SAT (Austin, 1962; Searle, 1976), speech acts are analyzed on the locutionary (i.e., actual utterance and its ostensible meaning), the illocutionary (i.e., propositional contents carrying intentions), and the perlocutionary (i.e., effect on the addressee) level.

In this paper, we adopt a semiotic approach to analyze pragmatics in human communication, based on Liu (2000). The concept of Pragmatics in OS is understood as the relations between the intentional use of a sign and its effects on people in a social context. According to Liu (2000), communication is successful when a meaningful sign is used with an appropriate intention between the speaker and the listener. Aligned with LAP and SAT, Liu's approach considers communication acts (or language acts) as the minimal unit of human communication. A complete communication act can be defined as a structure consisting of three components: performer, addressee, and message.

A message can be distinguished as having two parts:

1. The content part of a communication act manifests the meaning of the message. The meaning is determined by social construct or human behavior performed by the speaker and by the hearer. The semantics can also be obtained through indirect speech;

2. The function part of a communication act specifies the illocution which corresponds to the intention of the speaker.

Liu (2000) proposes to group the illocutions into three dimensions: time (i.e., whether the effect is on the future or the present/past), invention (i.e., if the illocution used in a communication act is inventive or instructive, it is called prescriptive, otherwise it is called descriptive), and mode (i.e., if it is related to expressing the personal modal state mood, such as feeling and judgment, then it is called affective, otherwise denotative). By using these dimensions, the illocutions are classified as:

1. Proposal (future, prescription and denotative);

2. Inducement (future, prescription and affective);

3. Forecast (future, description and denotative);

4. Wish (future, description and affective);

5. Palinode (present/past, prescription and denotative);

6. Contrition (present/past, prescription and affective);

7. Assertion (present/past, description and denotative);

8. Valuation (present/past, description and affective).

This classification can be associated with perlocutionary acts, and 
propositional attitudes that represent the effects on the addressee. The next section presents the empirical basis of this work, i.e. a previous study that explored possibilities of Pragmatics Communication Analysis to provide richer interaction mechanism for collaborative problem solving.

\section{Analyzing Intentions in Problem Solving}

The empirical data that has been analyzed in this work has been gathered during activities conducted in the context of a research project named "Social Networks and Professional Autonomy"3, which is in the domain of Webmediated continuous learning of Brazilian special education teachers. In the context of this project, the initial activities aimed at learning more about the way 28 participants use Web-based systems, and how they engage in different forms of Web-mediated conversations. To this end, four consecutive activities were conducted. Due to space limitations, the analysis presented in this work is based on two of the four activities, namely the discussions conducted in VilaNaRede and Yahoo! Answers ${ }^{\circledR}$. Yahoo! Answers ${ }^{\circledR}$ is a Web system that permits a user to post a question and other users to post answers to that question or vote for the best answer. Out of the 28 teachers, 16 participated in the two activities (15 in Yahoo! Answers ${ }^{\circledR}$ and 10 in VilaNaRede). These teachers had no previous experience with the two systems, but they were already used to Web applications such as blogs, email, and forums. A so called "case" (a problem related to the integration of a student with special needs in regular classes) was posted in the system and teachers were asked to discuss and solve it.

The content analysis was performed in three steps (Bonacin et al., 2012): the first step was related to a quantitative analysis of the interactions (posts); the second step involved the examination of messages using the pragmatic communication analysis; and the third synthesized the results and explored interaction possibilities based on the outcomes.

For each message, two analysts attributed continuous values from 0.0 to 1.0 for each dimension, e.g. 0.0 for a message that (s)he judged to be completely denotative, and 1.0 for a completely affective one. The main reasons for nondiscrete classifications are a phrase's ambiguity and intelligibility.

\footnotetext{
${ }^{3}$ www.nied.unicamp.br/tnr
} 


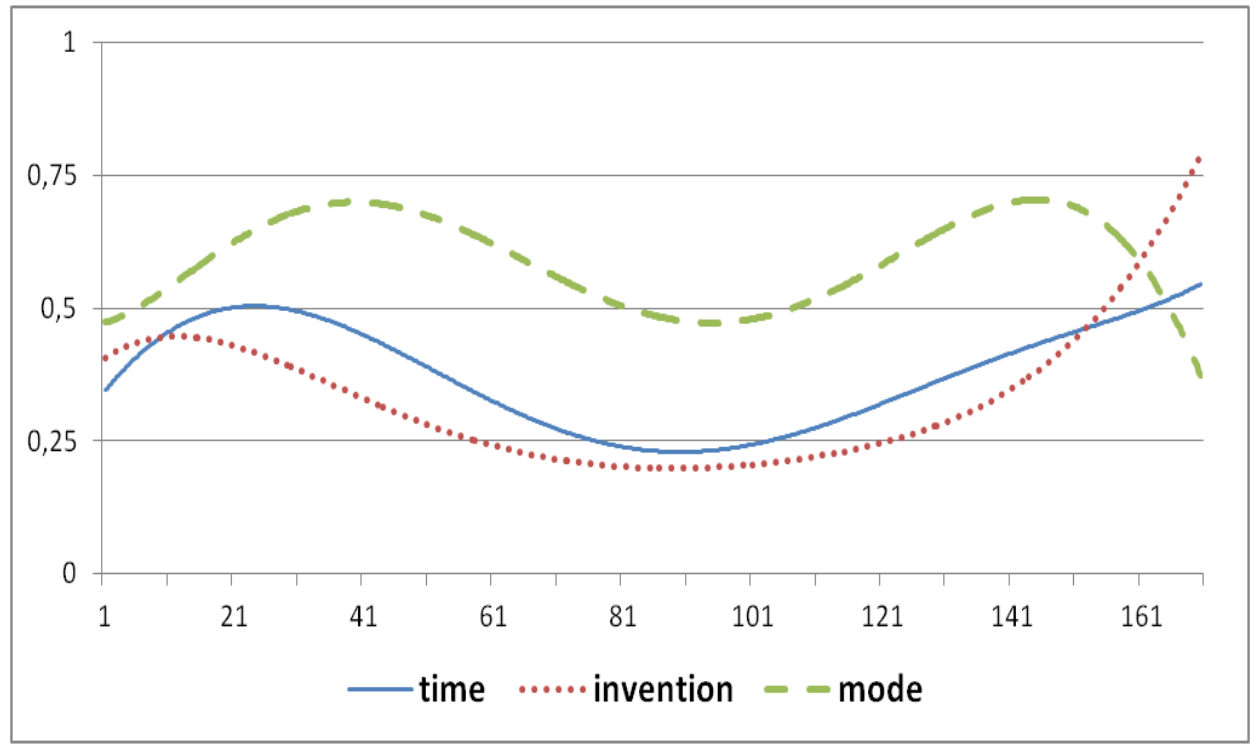

Figure 1. Analysis of the VilaNaRede case study

Based on the attributed values, a predominant classification was identified for each message, and optionally the analyst could also indicate a secondary (or alternative) classification. Furthermore, the analysts identified aspects associated with the content part: the role of who performed the message ("speaker"), and the main behavior pattern (identified as terms) that the message referred to.

A total of 170 communication acts were analyzed in the VilaNaRede case study. Fig. 1 presents the polynomial trend lines of the three dimensions (i.e., time, invention and mode) of the VilaNaRede case. Fig. 2, respectively, presents the polynomial trend lines of the Yahoo! Answers ${ }^{\circledR}$ case study, where a sequence of 318 communication acts was analyzed. In Fig. 1 and Fig. 2 the vertical axes represent the respective dimension values attributed to each communication message. Looking at the trend line we can visualize the evolution of the dimensions in the problem solving process. For instance, observing the invention dimension in Fig. 1, in the interval 61 to 101, there is a predominance of descriptive messages, while after message 151, the prescriptive messages predominate. 


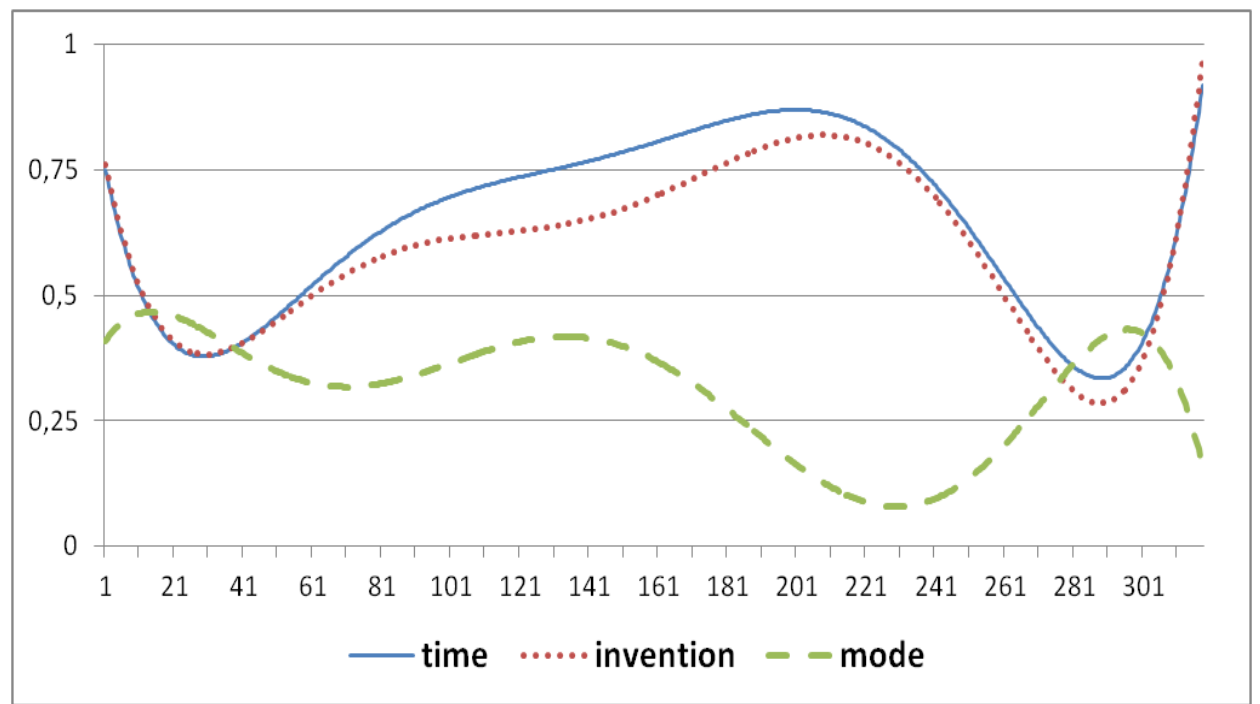

Figure 2. Analysis of the Yahoo! Answers ${ }^{\circledR}$ case study

From this analysis (Bonacin et al, 2012), a set of new possibilities to be explored was identified including:

1. identification of clusters of messages with the same predominant illocutions and subjects (e.g., valuations about the case discussion in the beginning of VilaNaRede case, as well as prescriptions of an attendance plan at the end of Yahoo! Answers case);

2. a more refined classification of the messages and the users' participation is also possible (e.g., to recover sequences of messages and who valuated/judged one specific alternative);

3. use of illocutions as an additional parameter in the semantic disambiguation process;

4. illocutions as palinodes and contritions with impacts on the interpretation of the discussion;

5. identification of issues that are not related to the main topics; among others.

\section{Modeling Meanings and Intentions}

Based on the case studies presented in the last section, we propose an ontology for representing pragmatic aspects in messages of collaborative problem solving systems. To this end, we describe the main concepts of the Pragmatics Communication Analysis in an OWL ontology. This allows correlating the representation of communication acts with external ontologies. Section IV.A. details the proposed ontology, and Section IV.B. presents a small example of a query over it. 


\section{A. The $\mathrm{CactO}$ - Communication act Ontology}

The $C a c t O$ was constructed in OWL using the Protégé tool ${ }^{4}$. Fig. 3 presents an overview of the modeled ontology with the main classes, their hierarchy, dataproperties and objectproperties. This figure also illustrates relations with user posts (in collaborative systems), and with external ontologies. The ontology has three main classes: Agent (who performs an act), Act (the performed action), and Behaviour_Pattern (the pattern that delineates the actions performed by one agent including the meaning interpretations). The class HumanAgent represents one specific person or a group of persons. A CommunicationAct is a subclass of Act. It has associations with two HumanAgents (the performer (hasPerformer) and the addressee (hasAddressee)) and with a MessageAct (hasMessage). The instances of the CommunicationAct are extracted from and are related to the content of collaborative systems, including who performs the message and for whom it is addressed.

The user posts are broken down into fragments that represent single CommunicationActs as the minimal units of communication. These fragments are associated with the properties of the CommunicationActs: the performer who created the post, the addressee to whom this specific fragment is addressed, and the message, i.e., the fragment itself. The Dataproperties that link the instances to the comment fragments were omitted in Fig. 3 to increase the readability of the figure. The cardinalities in the model were also omitted in the figure. The Dataproperties of CommunicationAct represent: the system_id, the post_id, as well the "start index" and "end index" of the fragment with respect to the complete post.

The MessageAct is a subclass of Act. It is composed by the content part (hasBehaviour_Pattern), and its functional part (illocution). To represent its functional part, the MessageAct class has four main Dataproperties: the illocution to set one as the group proposed in the classification of illocutions, and float values that represent the three dimensions of the illocution (i.e., time, mode and invention). Internally, the illocution is derived from the dimensions.

The MessageAct class has also two Objectproperties: hasBehaviour_Pattern links the message to a Behaviour_Pattern (i.e., the meaning of the message), and hasIndirectAct links this message to other instances of CommunicationAct in case of indirect acts. The latter is important to provide relations between different instances of CommunicationAct, where the meaning of one communication act is determined by another communication act (see indirect speech acts in (Searle, 1976)).

A Behaviour_Pattern is linked to concepts of external ontologies (or vocabulary sources) that have the same (owl:sameAs) meaning of the interpretation determined by the pattern of behavior. For instance, regarding the message "The virus is not eliminated from the body, and can cause shingles", it is expected to have a behavior of the agent towards the interpretation of "shingles"

\footnotetext{
${ }^{4}$ http://protege.stanford.edu
} 
as a disease. This behavior pattern can be linked, for instance, to the code B02 on the International Classification of Diseases (ICD-10 ${ }^{5}$ ). In contrast, the same term "shingles" is related to other behavior patterns in the message "How much does it cost to repair 3 shingles?", which can be linked to an external ontology/vocabulary relative to "roof" (or "construction").

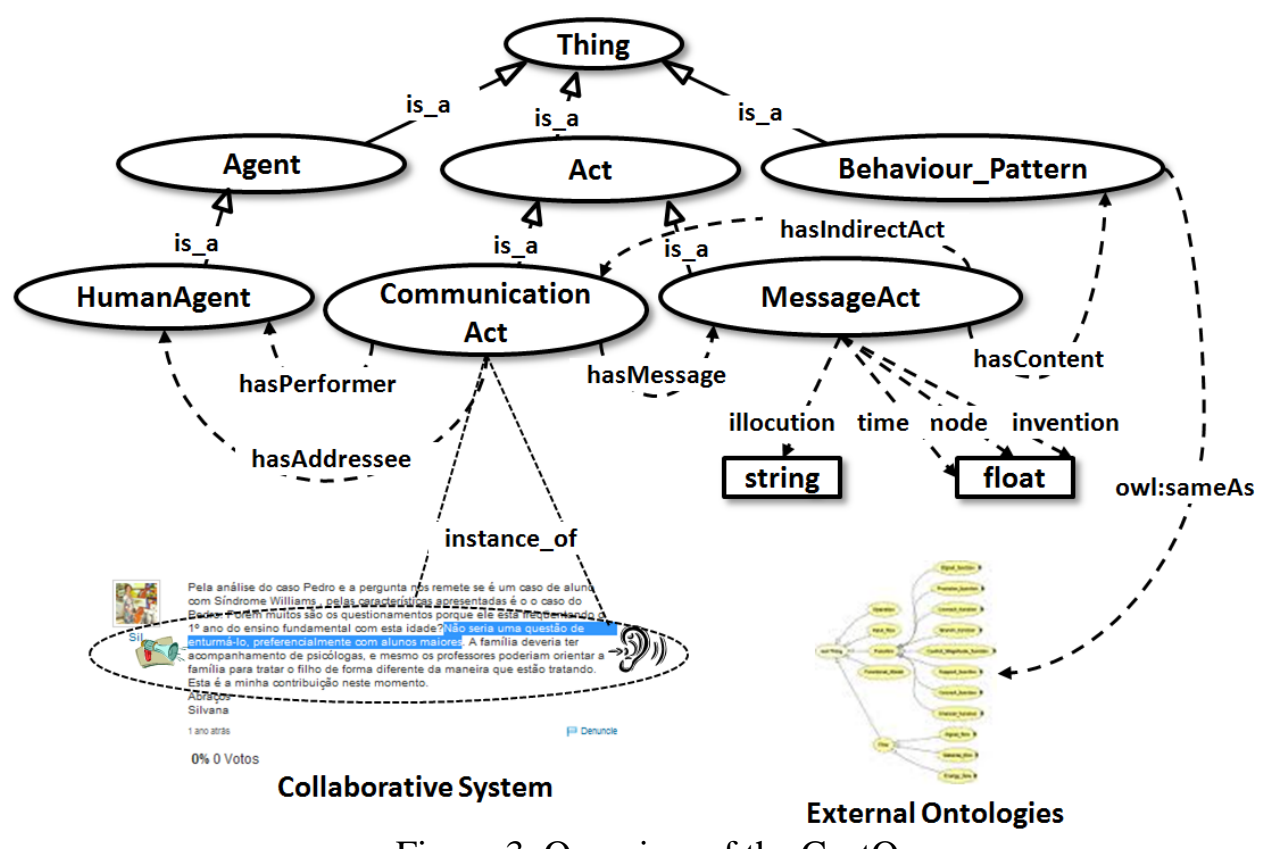

Figure 3. Overview of the CactO

It is possible to envisage various scenarios of applications for the proposed model. For instance, to understand and analyze a discussion, or to provide different alternatives or possibilities to recover messages taking into account the illocutions. In the following, we present a small example to illustrate the model.

\section{B. The CactO model in use}

Fig. 4 presents an example of a communication act modeled in the proposed ontology. The text fragment "I hope to learn a lot from this case" was extracted from the VilaNaRede case. This communication act was performed by one human agent (uid_30) and addressed to all users. The communication act has a message with a functional part that includes an illocution classified as a wish, based on the values of the three dimensions time, mode and invention. The content part is associated with behavior patterns that can be related to external concept definitions. In this example, the use of the word "case" in the act is related to a pattern towards the interpretation of the word "case" as the "case

\footnotetext{
${ }^{5}$ www.who.int/classifications/icd/en
} 
under study" in the problem solving processes. This behavior pattern might be associated to external sources that semantically define the word "case" as an instance of a problem, in opposition to the word "case" as a container. Instances of the model were generated from the analysis described in section III.

The instances of the CommunicationAct class were enumerated using the sequence numbers of the analyzed illocutions to facilitate visualization (the class has Dataproperties for a precise identification). For example, the "CommunicationAct_l" corresponds to the first illocution and the "CommunicationAct_318" corresponds to the illocution number 318 in the problem solving case.

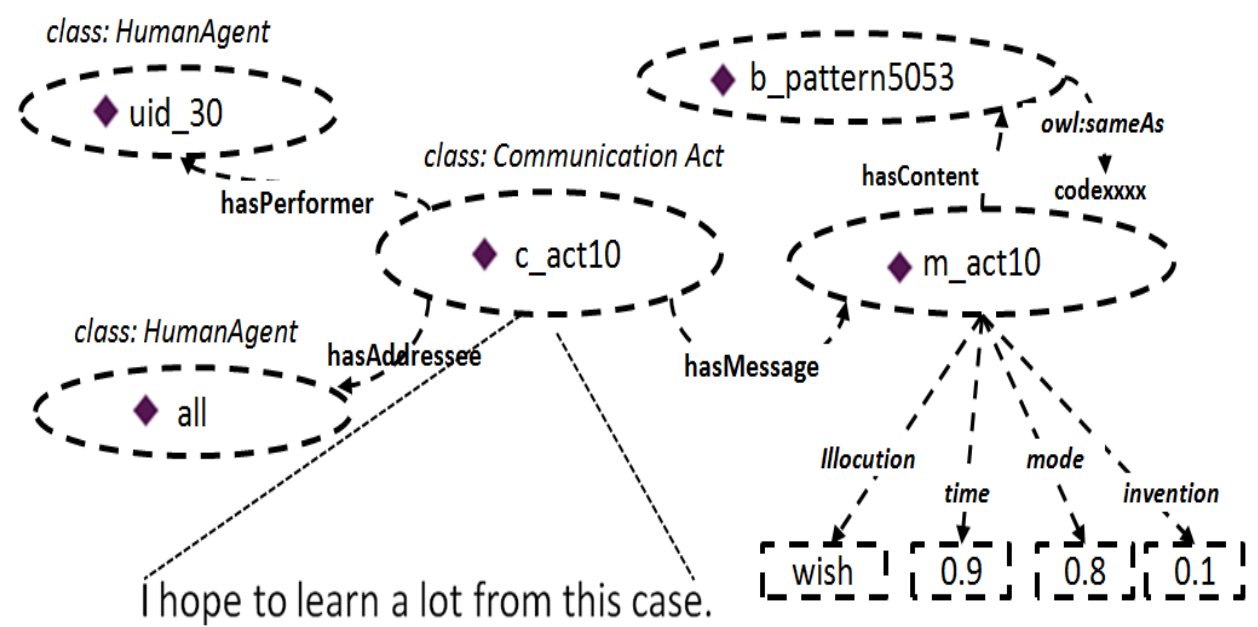

Figure 4. Example of an instance of CommunicationAct

We provide an example of an information retrieval scenario in order to illustrate how the model can be used for this purpose, and thus for enhancing information sharing in the collaborative problem resolution process. Fig. 5 presents a query using the SQWRLQueryTab ${ }^{6}$ for a scenario in the Yahoo! Answers ${ }^{\circledR}$ case (i.e., a student with William's syndrome). In this scenario, a conceivable retrieval scenario is "recover all the proposals regarding the student's family discussed in the case". The application of this query returned the total of sixteen communication acts with the "proposal" illocution and the behavior pattern "family", including: CommunicationAct_5, CommunicationAct_122, CommunicationAct_128, and Communication_Act_140.

As an example, in the CommunicationAct_122, one expert said: "The parents should also be informed of how to interact with Pedro, since he is a teenager". A simple lexical search would not be able to retrieve this communication act, since the word "family" is not explicitly written in the phrase, whereas a typical semantic search engine would be able to return this phrase since "parents" is part of "family". However, the pragmatic model also considered the illocutions which

\footnotetext{
${ }^{6}$ protege.cim3.net/cgi-bin/wiki.pl?SQWRLQueryTab
} 
reflect the intentions in order to distinguish the communication acts that are classified as proposals in the set of communication acts.

The proposed classification of messages might facilitate a more refined search, since it is possible to include intentional parameters in the search. That way, it would be possible to, e.g., distinguish specific acts with the objective of making proposals from valuations (Communication Acts 22, 89, 181, 213 and 265), assertions (Communication Acts 253, 254 and 263), etc. The parameters could also be combined to produce more complex queries or to include other predicates in the query, for instance, concerning the human agents.

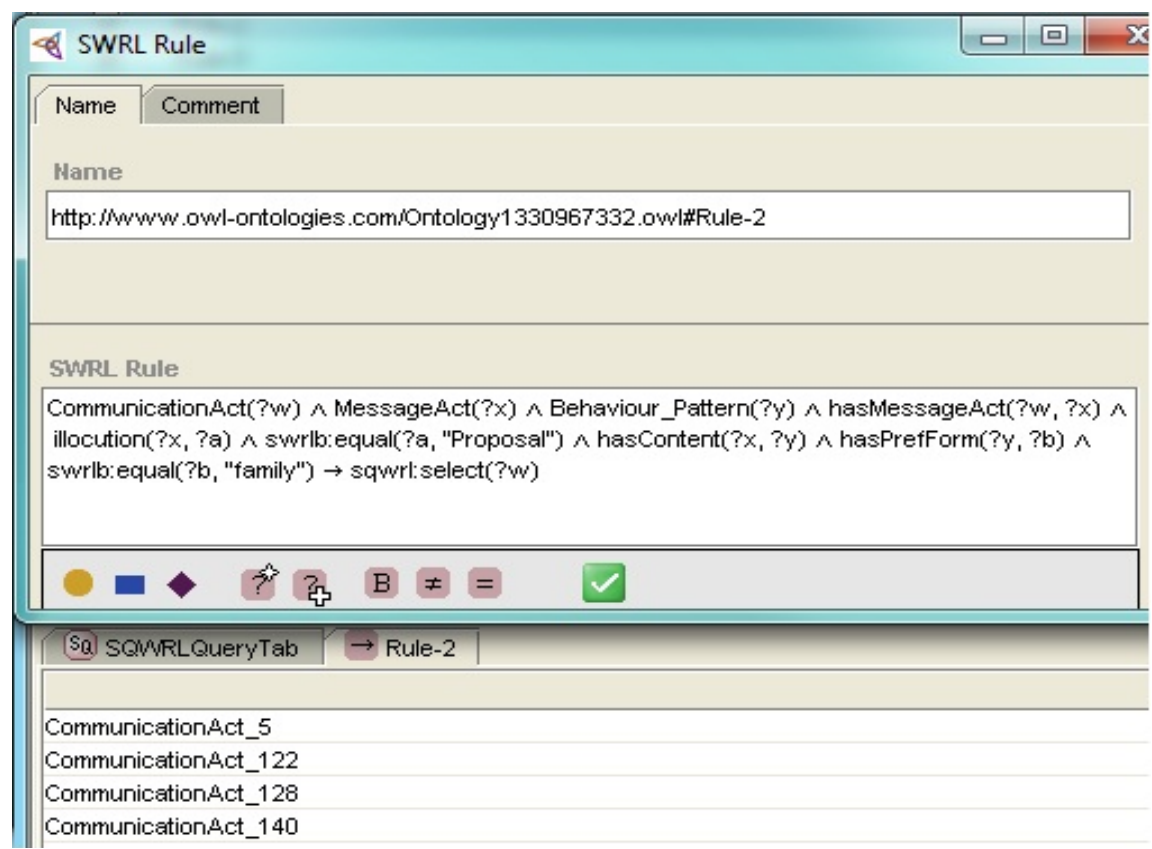

Figure 5. Example of query using SQWRLQueryTab

The retrieval of information based on pragmatic aspects might, for instance, be used to better understand the involvement of users in the problem under discussion. Moreover, other possible applications of the proposed model include the use in system design or evaluation (e.g., for evaluating how different systems or different iterations of the same system support certain conversational features), or the use within communities of practice to review how a conversation had developed, what principal difficulties were encountered, etc. Also, by using OWL, even more complex rules can be written enabling "cross ontology"-queries and semantic inferences.

\section{Illocution-based Message Retrieval}

In this section we present a laboratory study concerning the use of the CactO 
to support the retrieval of messages. The objective is to show the possibilities and limitations of the approach i.e., perspectives and research challenges. Note that it is out of the scope of this paper to present an extensive evaluation with hundreds (or even thousands) of users.

With this in mind, we propose retrieval scenarios exploring different illocutionary aspects. The objective is to analyze queries that might be relevant to users, analysts or other stakeholders. We aim at exploring situations where users might have benefits of new information sharing possibilities by using the proposed model. We observed whether these queries could be answered by $\mathrm{CactO}$, in addition we assessed the results of $\mathrm{CactO}$ by comparing them with other strategies of information retrieval, which do not consider the pragmatic aspects under investigation. The study evaluated the adequacy of the proposed model for representing intentions as identified by Bonacin et al. (2012). In the following we describe the materials, the methods and the results.

Materials. In this evaluation we used the CactO within the Protégé tool. An "external ontology" (as shown in Fig. 3) with the main concepts of the domain was also created in Protégé. The most relevant classes include: Case (related to the case descriptions), Children (related to the child/teenage behavior), Discussion (related to the discussion itself), Environment (related to environment conditions), Family (related to the student's family), Resources (related to the existing resources or needs for resources), School (related to the school conditions), Condition (related to the physical or psychological condition of the student under discussion), Solution (related to a solution for the case), System (related to the system or computational resources used in the discussion) and Teacher (related to teacher behavior or capabilities). Each class was detailed in is_a relations (hierarchical structure), part_of relations (part hierarchy) and same_as relations (synonyms). For example, Parents is_part of the family and Williams-Beuren syndrome is_a Condition. This ontology was used in both the message classification with $\mathrm{CactO}$ and without it to evaluate "simple" semantic search scenarios.

Content data from the case studies presented in Section III were utilized to create instances in the model and to respond to the retrieval scenarios. Protégé was used to manually create the instances. Queries were implemented using the SQWRLQueryTab Protégé plugin. The content of data was also used to compare the results of queries with the expected results.

Methods. After modeling the ontologies (i.e., CactO and the "external ontology"), we created instances of classes populating the model based on the categorization of communication acts performed in the case studies presented in Section III. Then, one Interaction Design specialist proposed in natural language scenarios related to a user's intentions. A total of eleven scenarios were described to identify situations where the representation of pragmatic aspects might provide better search results or classification from the content of the collaborative problem system. Two of them are deeper analyzed and presented in the 
following. Generally, the scenarios explored aspects such as: identification of predominant illocutions and subjects, the use of more refined classifications considering illocutions, the use of illocution classification for disambiguation, and filtering "off-topic" messages. The scenarios studied are:

- Scenario 1: Get all proposals and valuations regarding the involvement of the student's family;

- Scenario 2: For each forecast or wish regarding a topic, get related messages, i.e., messages about the topic that were created after the forecast/wish;

Based on the scenarios we performed four types of queries:

- The first exploring the $\mathrm{CactO}$ with the external model $\left(\mathrm{S}_{\text {cacto }}\right)$;

- The second as a "simple" semantic search using the class index based on the "external model" $\left(\mathrm{S}_{\text {sem }}\right)$, i.e., the same model used with CactO. The objective was to "calibrate" the class index in both cases $\left(\mathrm{S}_{\text {cacto }}\right.$ and $\mathrm{S}_{\mathrm{sem}}$ );

- The third as a syntactic search just using word matching $\left(S_{\text {syn }}\right)$. Lexical variations, derived words, and common typing mistakes were also considered in the search;

- The fourth as a manual content analysis $\left(S_{\text {man }}\right)$, which was used to evaluated the other three query types.

To avoid biased evaluation results, the same specialist who suggested the search scenarios also performed the manual content analysis, while one of the other authors implemented and executed the other three types of queries.

For each scenario, we collected the results for the four types of search and evaluated the precision $(\mathrm{P})$ and recall $(\mathrm{R})$. These measures were used to analyze the search effectiveness by indicating the fraction of retrieved instances that are relevant (precision), and the fraction of relevant instances that are retrieved (recall). The precision of the $S_{\text {cacto }}$ is determined by:

$$
\mathrm{P}_{\text {cacto }}=\left|\left\{\mathrm{S}_{\mathrm{man}}\right\} \cap\left\{\mathrm{S}_{\text {cacto }}\right\}\right| /\left|\left\{\mathrm{S}_{\text {cacto }}\right\}\right|
$$
and the recall $R_{\text {cacto }}$ is determined by:

$$
\mathrm{R}_{\text {cacto }}=\left|\left\{\mathrm{S}_{\text {man }}\right\} \cap\left\{\mathrm{S}_{\text {cacto }}\right\}\right| /\left|\left\{\mathrm{S}_{\text {man }}\right\}\right|
$$

The $\mathrm{P}_{\mathrm{sem}}, \mathrm{R}_{\mathrm{sem}}, \mathrm{P}_{\mathrm{syn}}$, and $\mathrm{R}_{\mathrm{syn}}$ were determined using the same formulas.

A final analysis involved a qualitative analysis that aimed at understanding why certain results did or did not appear in the result sets of $S_{\text {cacto, }} S_{\text {sem, }}$ and $S_{\text {syn. }}$.

Results. The proposed scenarios in this evaluation illustrate possible applications and benefits of the proposed model, as well as the importance of considering pragmatic aspects in message retrieval. It is not expected to produce results for comparing the performance of the approaches.

\section{Quantitative results of Scenario 1:}


- CactO: $\mathrm{P}_{\text {cacto }}=1.0$, this means that in this specific scenario all (27) the results of $S_{\text {cacto }}$ are also present in $S_{\text {man }}$. The $R_{\text {cacto }}=0,75$, this means (as the precision was 1.0) that $S_{\text {cacto }}$ returned $75 \%$ of the messages (27 of 36) detected in $\mathrm{S}_{\mathrm{man}}$;

- Semantic Search: $\mathrm{P}_{\mathrm{sem}}=0.79$, this means that $79 \%$ of the results of $S_{\text {sem }}\left(27\right.$ of 34) are also present in $S_{\text {man }}$. The $R_{\text {sem }}$ was the same of $R$ cacto, that is $75 \%$ (27 of 36 ).

- Syntactic Search: $\mathrm{P}_{\mathrm{syn}}=0.70$, this means that $70 \%$ of the results of $\mathrm{S}_{\mathrm{syn}}$ (26 of 37) are also present in $S_{\text {man. }}$ The $R_{\text {syn }}=0.72$, that means $R_{\text {syn }}$ returned $72 \%$ of the messages ( 26 of 36 ) detected in $S_{\text {man }}$.

Fig. 6 illustrates a chart with Precision and Recall for the Scenario 1 concerning the three non-manual types of search.

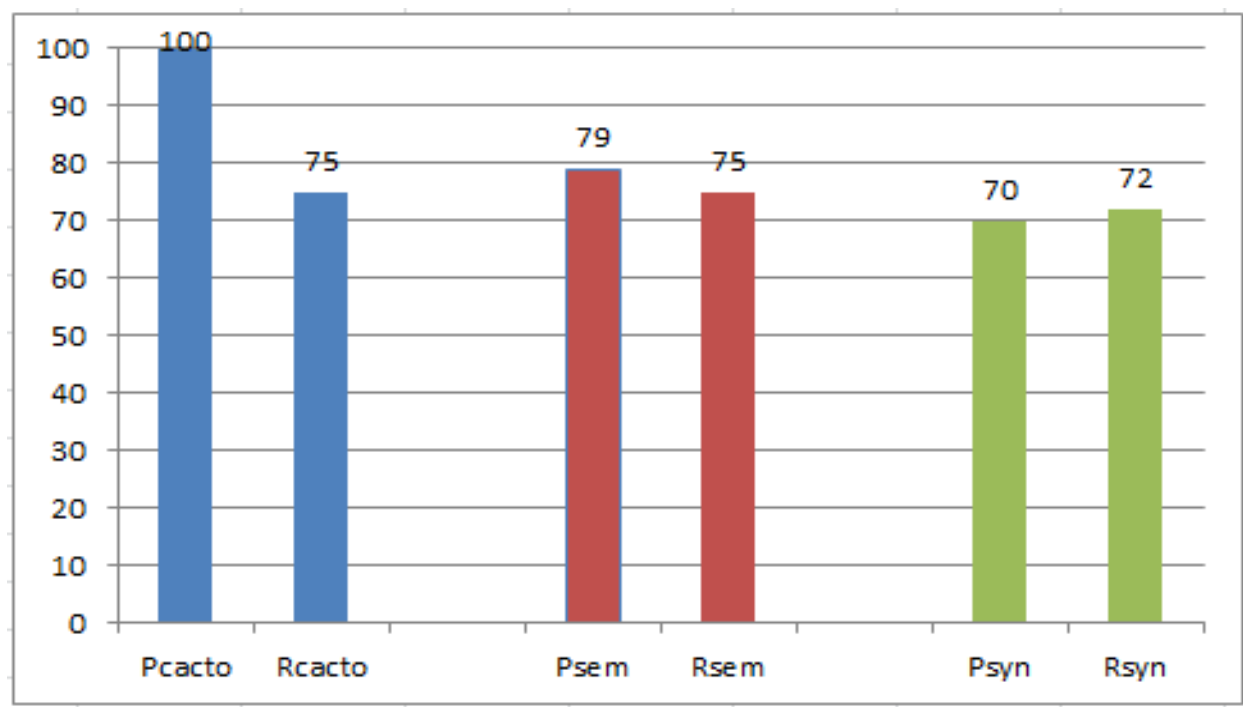

Figure 6. Scenario 1: Precision and Recall of the non-manual search types in $\%$

\section{Quantitative results of Scenario 2:}

- CactO: $\mathrm{P}_{\text {cacto }}=0.29$, this means that $29 \%$ of $\mathrm{S}_{\text {cacto }}(18$ of 63$)$ are also present in $S_{\text {man }}$. The $R_{\text {cacto }}=1.0$, this means that $S_{\text {cacto }}$ returned $100 \%$ of the messages (18) detected in $S_{\operatorname{man}}$;

- Semantic Search: $\mathrm{P}_{\mathrm{sem}}=0.11$, this means that $11 \%$ of the results of $S_{\text {sem }}$ (18 of 169) are also present in $S_{\text {man }}$. The $R_{\text {sem }}$ was the same of $\mathrm{R}_{\text {cacto, }}$, that is $100 \%$ (18).

- Syntactic Search: $\mathrm{P}_{\mathrm{syn}}=0.07$, this means that $7 \%$ of the results of $\mathrm{S}_{\mathrm{syn}}$ (1 of 15 ) are also present in $S_{\text {man. }}$ The $R_{\text {syn }}=0.06$, that means $R_{\text {syn }}$ returned $6 \%$ of the messages ( 1 of 18 ) detected in $S_{\operatorname{man}}$.

Fig. 7 illustrates a chart with Precision and Recall for the Scenario 2 concerning the three types of search. 


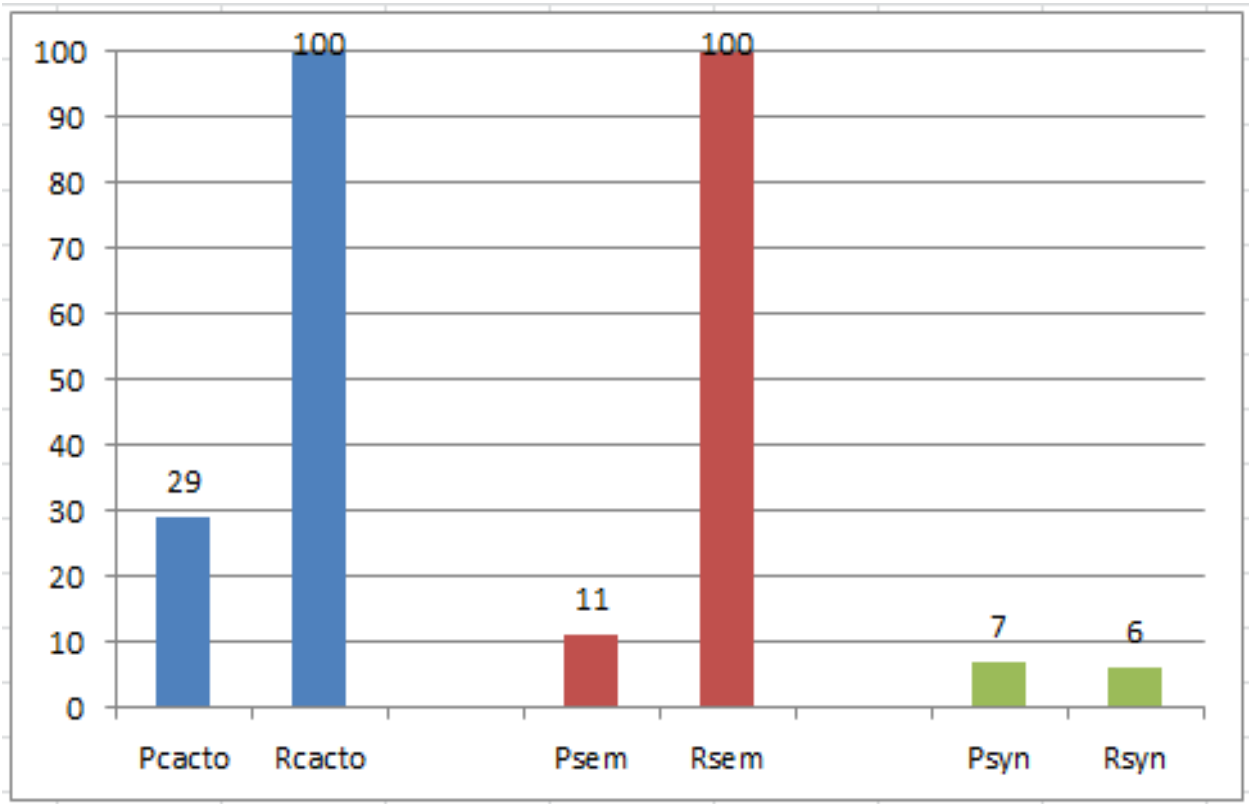

Figure 7. Scenario 2: Precision and Recall of the non-manual search types in \%

\section{Qualitative Analysis of the Results:}

We present a qualitative analysis of the results organizing them by different strategies. First, we present an analysis independent of scenarios or queries. Subsequently, we provide an analysis independent of search types. Finally, an analysis focused on each search type is presented.

\section{Analysis independent of scenario or query}

If we assume that the queried data is always coded or annotated "correctly", the way the search types are defined in this paper $S_{\text {cacto }}$ will always contain a subset of $\mathrm{S}_{\text {sem }}$. Consequently, the $\mathrm{R}$ (recall) of $\mathrm{S}_{\text {cacto }}$ will be never higher than $S_{\text {sem. }}$. In fact, $S_{\text {cacto }}$ represents a more refined classification in a previously classified text. Therefore, a better $\mathrm{P}$ (precision) of $\mathrm{S}_{\text {cacto }}$ was expected and confirmed in the scenarios studied (i.e., in both cases $\mathrm{P}_{\text {cacto }}$ was higher than $\mathrm{P}_{\text {sem }}$ ).

Although the studies have a limited statistical basis (e.g., few scenarios considered), we can say that compared to $S_{\text {cacto }}, S_{\text {sem }}$ and $S_{\text {syn }}$ are likely to have an inferior $\mathrm{P}$, if the query contains a clause that restricts the selection of illocution types. The $\mathrm{P}$ will decrease with an increasing restrictiveness of this clause. Besides the clause itself (e.g., the number of illocution types queried), the restrictiveness also depends on the distribution of the search space (queried data). For example, in the Yahoo! Answers ${ }^{\circledR}$ case, which is characterized by a high number of proposals, a query for inducements would be very restrictive and thus the $\mathrm{P}$ of $\mathrm{S}_{\mathrm{sem}}$ and $\mathrm{S}_{\mathrm{syn}}$ would be relatively low.

Note that although $S_{\text {cacto }}$ presented the highest Precision and Recall, there might be situations where this does not occur. In practice, we cannot assume that 
Title

all the illocutions and messages relations were correctly identified and codified (even when this task was completely performed by humans). First, coding depends on subjective factors, e.g., one person might interpret a message as a response to another message, while another person might interpret the two messages as independent of each other. Second, a computational implementation would thus rely upon rules or other concepts that reflect this subjectivity, and therefore would use heuristics or other non-deterministic mechanisms. As a consequence, the Precision and Recall of $S_{\text {cacto }}$ might be inferior to $S_{\text {sem }}$ or $S_{\text {syn }}$ in some specific situations.

\section{Analysis independent of search type}

The queries of a user frequently are of a complex nature, which provides various difficulties regarding how to analyze the expected results. The queries leave room for interpretation and ambiguities since they depend on the user's pragmatic context which is usually different from the pragmatic contexts of the people who implemented the search mechanism. For example, "involvement of the family" might mean different things for different people. For some people it might mean "an active role of the family in the case resolution", while others might expect that a query using this term returns anything that mentions the family in an active or passive role. In other words, although Precision and Recall suggest some objective measure of the quality of the result sets, this quality ultimately depends on the assessment of the person requesting the query. The evaluation of search results entails various research challenges.

In practice, this means it is usually difficult to achieve absolute Precision and Recall measures. Hence, compromises have to be made, e.g., the query terms could be relaxed, yielding higher Recall and lower Precision. Further investigations are required to better understand these effects and how query terms could be combined. Moreover, it is not always necessary to rely on a unique type of search. It is conceivable to combine $S_{\text {cacto }}$ with $S_{\text {sem }}$ or $S_{\text {syn }}$ depending on query complexity. The analysis of the domain and purpose of the collaboration could also be taken into account, e.g., the context and domain of the shared work practices may bring some extra parameters to increase the Precision. However, how to conduct the definition of these parameters requires additional investigation.

\section{Analysis of CactO}

Queries that result in smaller Precision or Recall usually involve complex clauses regarding relations between illocution types. For example, if Scenario 1 changed to "Get all valuations of proposals ...", messages would have to be coded accordingly to reflect these relations. As a workaround, users could relax the clause to "Get all valuations and proposals ..." yielding a lower Precision and manually select the correct results. In Scenario 2 the value of $P_{\text {cacto }}$ was much lower than in Scenario 1. One reason is the existence of rhetoric messages that 
were retrieved by the query. For example, in Scenario 2, one message classified as wish was rhetoric in nature ("I hope to have contributed with this comment") and referred to message acts before the actual wish, which $S_{\text {cacto }}$ is not able to retrieve at the moment. Another element that resulted in a lower $\mathrm{P}$ of $\mathrm{S}_{\text {cacto }}$ in Scenario 2 is the fact that the case studied in the two scenarios was purely hypothetic. Thus, forecasts and wishes regarding the "student" had no consequences. $S_{\text {cacto }}$ is not able to represent differences between substantial and hypothetic/rhetoric messages.

\section{Analysis of the Semantic Search}

Messages that were not returned by the $\mathrm{S}_{\text {sem }}$ (i.e., resulting in a lower Recall for $S_{\text {sem }}$ and consequently for $S_{\text {cacto }}$ as well) are those where the content is not directly (or obviously) related to the content part of the query. For example, regarding Scenario 1, the message "does he sleep well?" is indirectly related to the student's life at home, and thus to the involvement of the family. Other messages made a reference to "the other involved parties" and listed the family as an involved part in previous messages. In these cases the meaning is determined by a very dynamic context of use, instead well-defined and static domain semantics.

In order to be able to retrieve those messages, a textual preprocessing would be required, which could be costly and complex, since it is conceivable that not only each newly created message would have to be preprocessed (e.g., link "involved parties" to "family" and others), but also that the existing message corpus or index would have to be reprocessed, for instance, if new terms are introduced for existing concepts or if meanings of existing concepts change over time. Such evolutionary aspects in ontologies are open research issues in SemWeb. They might play a role in influencing Precision and Recall of $S_{\text {sem }}$ and $S_{\text {cacto }}$.

\section{Analysis of the Syntactic Search}

$\mathrm{S}_{\mathrm{syn}}$ returned some typical results that illustrate shortcomings of purely syntactic search strategies. For example, in Scenario 1 it returned results where participants suggested that the student under discussion should make drawings of family members. In queries that use very complex clauses regarding relations between illocution types, $S_{\text {syn }}$ might yield higher Recall, probably at the cost of lower P, simply due to the fact that it does not handle illocution types at all, and thus always performs relaxed queries that return more results. On the other hand, queries that use only illocution types and no message content (e.g., "get all forecasts") require additional effort from the user (e.g., a search for lexical variations of "bet", "predict", "I'm sure that ... will ...", etc.). As expected, $S_{\text {syn }}$ had lower $R$ than $S_{\text {sem }}$ and $S_{\text {cacto }}$ caused mainly by cases where a variety of synonymous were used to express the same concept, as well as when the concepts were expressed in multiples abstraction or detailing levels. 
The following section discusses strengths and limitations of the approach considering the results analyzed in this section. Related work and unaddressed research challenges are also presented.

\section{Discussion}

In this paper, we presented a model to represent some crucial pragmatic aspects of communication acts using SemWeb technologies. The studies presented in Section III and scenarios of the previous section have demonstrated the possibilities of a model as proposed in this investigation. However, they have also brought up theoretical and practical limitations of CactO. For instance, the explored dimensions are able to represent only a subset of pragmatic aspects, and aspects such as propositional attitudes are still not considered in the model (e.g., the intention of the speaker might not be understood by the addressee, or it might be understood, but not result in the response intended by the speaker).

Propositional attitudes refer to the mental states of speakers and addressees. The affective (like-dislike and desire-fear) and denotative (belief-disbelief) scales can express important aspects of the speakers' and addressees' mental states. The propositional attitudes may also be investigated through an analysis of the perlocutionary acts. The analysis of these acts imposes additional difficulties compared with the investigation presented in this paper, such as the identification of the propositional attitudes of the speaker and addressee before and after the locution. Liu (2000) presents a correlation of the expected propositional attitude change and the illocution classes. This can be a starting point for future research towards more sophisticated pragmatic representations and their better exploration. One limitation of Liu's model that has to be overcome is that it focuses only on communication acts performed in an honest, sincere and reliable manner, and assumes that the speaker's intention is expressed clearly in the communication act. However, in the empirical studies we found that in some cases, meaning was not simply shared by uttering objective facts. Thus, Liu's assumption seems to be limiting, although necessary in order to be able to create "computable" representations. However, acknowledging the fact that Liu's assumption does not always hold, it becomes clear that these representations are approximations to the meanings constructed by collaborators and observers. In cases where meaning has to be constructed and negotiated, rhetoric is an important aspect of communication, and intentions might not be clearly expressed in the speech act. This is even truer when speaker and addressee do not know each other well, as is the case of the studies described in this paper. Nevertheless, this kind of problem was minimized in the scenarios analyzed due to the nature of the collaboration. The participants shared work practices using a common vocabulary in formal discussion in the most of the messages.

Although some works in SemWeb have tried to represent and recognize intentions in digital environments during the last years (e.g., Goss et al., 1999), there is still no ontological model to precisely represent pragmatics in communication in a way that can be adequately applied to problem solving and collaborative systems. The Change and Annotation Ontology (ChAO) (Noy et 
al., 2006), for instance, enables to record changes and meta-information about changes during collaborative modeling. Intentions could be expressed through annotations. However, the $\mathrm{ChAO}$ has not been proposed to be a model to explicitly represent intentions in the collaborations.

Hoekstra (2010) proposes a design pattern for representing roles which are central concepts for describing social reality. This proposal relies on new features of OWL 2 for capturing the semantics of roles, intentional concepts and $n$-ary relations (Noy and Rector, 2006) as reification. In Hoekstra's proposal, abstractions are used over reified relations through the use of complex axioms. Although Hoekstra claims that his approach might be used for representing intentional categories (propositional attitudes such as beliefs and desires) as well as speech acts, the solution is not designed to support the representation of intentions in a problem solving context. In contrast, the model proposed in this paper is informed by communication theories grounded in pragmatics, which might more adequately support the analysis of intentions in communication. Moreover, the design patterns proposed by Hoekstra are not a full ontological model that represents the communication and the intentions of messages as an integrated representation. Design patterns such as those can be potentially useful to extend the model presented in this paper.

Other recent approaches in the literature aim to analyze the users' behavior in collaborative environments using SemWeb technologies. For example, Angeletou et al. (2011) proposed an approach to represent and compute behavior by inferring roles in online communities. They employ semantic rules to label community users with their role. Nevertheless, these behavior roles are not linked to pragmatic aspects such as intentionality.

Kanso et al. (2007) present an approach to model intentions by analyzing the authors' acts. Their work differs from this paper in many aspects, including:

- Domain and purpose: since their work focuses on detecting intentions in scientific documents;

- Technology: since our work uses OWL and SWRL to be a bridge to external ontologies;

- Conceptualization: since, although the works have common roots, the investigation presented here also includes a well defined theoretical semiotic basis for the presented problem.

Although the focus of this paper is on modeling and representation aspects, it is worth noting that from the practical point of view it is necessary to consider the (semi-)automatic detection and classification of the communication and message acts. The analysis described in Section III was performed by humans without computational support. As a time-consuming task, it is possible to perform this analysis for a small set of cases. However, a higher degree of automation and computational support is needed when we consider real systems with thousands or even millions of messages. The construction of such supporting tools depends on studies of text mining and automatic text classification techniques. They are crucial for the scalability of the proposed 
approach. Various techniques and tools for text mining, automatic text classification, and semantic extraction can be used along with the approach proposed in this paper. It is beyond the scope of this paper to go deeper into the analysis of these techniques and tools, as well as performance issues. Furthermore, the use of "tags" in this task may also be investigated, since they might complement or, in some simpler cases, even substitute the complete analysis of the texts.

The adequacy of this model for other contexts has also to be studied. The modeling of pragmatic aspects of the communication acts might bring multiple benefits to better explore the messages exchanged in the problem solving process in general. The means to construct these queries or to select them, and then visualize the results based on the content of the collaboration is also a topic to be explored in future investigations.

\section{Conclusion}

Collaborative problem solving processes demand intensive communication. In this context, the classification of messages using knowledge representation models is a crucial factor to retrieve meaningful information for users. Pragmatic aspects, such as intentions, have a large influence on how people interpret and make sense of the shared content. This is the main reason why pragmatics is an important issue to be taken into account when analyzing and retrieving the messages of collaborations in problem solving.

In this work, we presented an ontology model named $\mathrm{CactO}$. It was written in OWL and based on the Pragmatics Communication Analysis. Communication acts were classified in the proposed model according to the illocutions classification in three dimensions (i.e., time, invention and mode). Instances of the proposed model were created from two real case studies where education specialists share problems of their daily work activities. We illustrated practical examples from these case studies using the $\mathrm{CactO}$ model. Furthermore, we conducted an experimental evaluation exploring various retrieval scenarios in order to observe the capacities and limitations of the model to provide richer and more precise message classifications. The results showed the feasibility of the proposal and new information sharing possibilities brought by the model, as well as scientific and practical challenges to be addressed.

Future work includes the study of new methods and mechanisms for a semiautomatic classification of illocutions from the textual content. We aim to conduct an investigation of the adequacy of different techniques of Natural Language Processing for the detection and classification of illocutions and other pragmatic aspects. We envision the construction of user interfaces and interaction mechanisms that explore the model in use, such as visualizations of the search results. Future investigations also include the impacts of pragmatic aspects in the Interaction Design of Web applications for collaborative problem solving, as well as the refinement and a deeper validation of the proposed model based on (empirical) field studies. 
Author

\section{Acknowledgment}

This work is partially funded by CNPq (\#560044/2010-0, \#141058/2010-2), by Proesp/CAPES (\#23038.01457/2009-11), and CAPES (\#01-P-08503/2008). The authors also thank colleagues from InterHAD for insightful discussions.

\section{References}

Angeletou, S.; Rowe, M. Alani, H.'Modelling and Analysis of User Behaviour in Online Communities', The Semantic Web - ISWC 2011. Lecture Notes in Computer Science, pp. 3550, 2011.

Austin, J. L. How to Do Things With Words. Oxford University Press: Oxford, England, 1962.

Bonacin, R., Hornung, H., Dos Reis, J. C., Pereira, R., Baranauskas, M. C. C. 2012. 'Interacting with Dynamic Social Knowledge - Revealing Challenges through an Analysis of Pragmatic Aspects of Problem Solving', Proceedings of the 14th International Conference on Enterprise Information Systems, Volume 3. Wrocław, Poland, pp. 54-63.

Cordeiro, J. and Filipe, J. 'Language Action Perspective, Organizational Semiotics and the Theory of Organized Activity - A Comparison', Proceedings of the workshop DEMO. Tilburg, the Netherlands, 2003.

de Moor, A. and van den Heuvel, W.J.. 'Web Service Selection in Virtual Communities', In HICSS '04: Proceedings of the 37th Annual Hawaii International Conference on System Sciences Track 7, Washington, DC, USA. IEEE Computer Society, 2004.

Gibson,J. J. The Ecological Approach to Visual Perception. Houghton Miffin Company, Boston, Massachusetts, 1968, 127-143.

Goldkuhl, G., and Lyytinen, K. 'A Language Action View of Information Systems', Proceedings of the 3rd International Conference on Information Systems, M. Ginzberg and C. Ross (eds.), Ann Arbor, MI, pp. 13-29, 1982.

Goss, S., Heinze, C. A., and Pearce, 'A. Recognising User Intentions in a Virtual Environment', Proceedings of the Simulation Technology and Training Conference (SimTecT), Melbourne, pages 247-254, 1999.

Hendler, J. \& Berners-Lee, T.. 'From the Semantic Web to Social Machines: A research challenge for AI on the World Wide Web', Artificial Intelligence. Elsevier, 2010, 174, pp.156-161.

Hoekstra, R. 'Representing social reality in OWL 2', Proceedings of OWLED, 2010.

Hornung, H. and Baranauskas, M. C. C. 'Towards a Conceptual Framework for Interaction Design for the Pragmatic Web ', Proceedings of 14th International Conference on Human-Computer Interaction, 2011. Heidelberg : Springer, 2011, p. 72-81.

Kanso H., Soulé-Dupuy C., and Tazi S. 'Representing Author's Intentions of Scientific Documents', Proceedings of International Conference on Enterprise Information Systems 2007, Funchal, Pt, v. 3, 489-492, 2007

Liu, K. Semiotics in Information Systems Engineering. Cambridge University Press, New York, NY, USA, 2000.

Liu, K.. 'Pragmatic Computing - A Semiotic Perspective to Web Services', In Filipe, J. and Obaidat, M. S., editors, E-Business and Telecommunications. ICETE 2007, Revised Selected Papers, volume 23 of Communications in Computer and Information Science, pages 3-15. Springer, Berlin, Germany, 2009. 
Title

McCool, R. 'Rethinking the Semantic Web, Part 1', IEEE Internet Computing, Vol. 9, No. 6, pp. $86-88,2005$.

Morris ,C . W. 'Foundations of the theory of signs ', International Encyclopedia of Unified Science, 1 (2). University of Chicago Press, Chicago, 1938.

Noy, N. F., Chugh, A., Liu, E. and Musen, M. A. 'A Framework for Ontology Evolution in Collaborative Environments', Proceedings of 5th International Semantic Web Conference, Athens, GA., 2006.

Noy, N. and Rector, A. 'Defining n-ary relations on the semantic web', Proceedings of W3C Working Group Note, 2006.

Paschke, A., Boley, H., Kozlenkov, A., and Craig, B. 'Rule Responder: RuleML-Based Agents for Distributed Collaboration on the Pragmatic Web', Proceedings of ICPW '07: Proceedings of the 2nd International Conference on Pragmatic Web, pages 17-28, New York, NY, USA. ACM, 2007.

Schoop, M., de Moor, A., and Dietz, J. L. G.. 'The Pragmatic Web: A Manifesto', Communications of the ACM, 2006, 49(5):75-76.

Searle, J. R. 'A Classification of Illocutionary Acts', In Language in Society, 1976, Vol. 5, No. 1, pp. $1-23$

Singh, M. P.. 'The Pragmatic Web', IEEE Internet Computing, 2002 6(3):4-5.

Stamper, R. Signs, 'Information and Systems', in B. Holmqnist, et. Al. (Eds) Signs of Work Semiotics Information Processing in Organisations, Walter de Gruyter, N. Y., 1996.

Winograd, T. and Flores, F. Understanding Computers and Cognition: A New Foundation for Design, Addison-Wesley Longman Publishing Co., Inc., Boston, MA, USA, 1987. 